\title{
Transcription factors expressed in soybean roots under drought stress
}

\author{
S.S. Pereira ${ }^{1}$, F.C.M. Guimarães ${ }^{2}$, J.F.C. Carvalho ${ }^{2}$, R. Stolf-Moreira ${ }^{3}$, \\ M.C.N. Oliveira ${ }^{2}$, A.A.P. Rolla ${ }^{3}$, J.R.B. Farias ${ }^{2}$, N. Neumaier ${ }^{2}$ and \\ A.L. Nepomuceno ${ }^{2}$ \\ 'Universidade Estadual de São Paulo "Julio de Mesquita Filho", \\ Jaboticabal, SP, Brasil \\ ${ }^{2}$ Embrapa Soja, Londrina, PR, Brasil \\ ${ }^{3}$ Universidade Estadual de Londrina, Londrina, PR, Brasil \\ Corresponding author: F.C.M. Guimarães \\ E-mail: francm@cnpso.embrapa.br
}

Genet. Mol. Res. 10 (4): 3689-3701 (2011)

Received May 5, 2011

Accepted September 22, 2011

Published October 21, 2011

DOI http://dx.doi.org/10.4238/2011.October.21.5

\begin{abstract}
To gain insight into stress-responsive gene regulation in soybean plants, we identified consensus sequences that could categorize the transcription factors MYBJ7, BZIP50, C2H2, and NAC2 as members of the gene families myb, bzip, c2h2, and nac, respectively. We also investigated the evolutionary relationship of these transcription factors and analyzed their expression levels under drought stress. The NCBI software was used to find the predicted amino acid sequences of the transcription factors, and the Clustal X software was used to align soybean and other plant species sequences. Phylogenetic trees were built using the Mega 4.1 software by neighbor joining and the degree of confidence test by Bootstrap. Expression level studies were carried out using hydroponic culture; the experiments were designed in completely randomized blocks with three repetitions. The blocks consisted of two genotypes, MG/ BR46 Conquista (drought-tolerant) and BR16 (drought-sensitive) and the treatments consisted of increasingly long dehydration
\end{abstract}


periods $(0,25,50,75$, and $100 \mathrm{~min})$. The transcription factors presented domains and/or conserved regions that characterized them as belonging to the bzip, c2h2, myb, and nac families. Based on the phylogenetic trees, it was found that the myb, bzip and nac genes are closely related to myb78, bzip 48 and nac2 of soybean and that $c 2 \mathrm{~h} 2$ is closely related to $c 2 h 2$ of Brassica napus. Expression of all genes was in general increased under drought stress in both genotypes. Major differences between genotypes were due to the lowering of the expression of the $m y b j 7$ and $c 2 h 2$ genes in the drought-tolerant variety at some times. Over-expression or silencing of some of these genes has the potential to increase stress tolerance.

Key words: Soybean; Drought stress tolerance; Transcription factor; Gene expression; Real-time PCR

\section{INTRODUCTION}

Environmental stress presents a major challenge in our quest for sustainable food production as it reduces the potential yields. Drought stress imparted by drought and temperature severity is the most prevalent abiotic stress that limits plant growth and productivity. In Brazil, the second world soybean producer, $20 \%$ of losses was due to water deficit, during the 2003-2004 crop seasons in the major producer states (Embrapa 2004; Conab 2005).

Although the number of genes known to moderate stress responses has increased (Kizis et al., 2001, Shinozaki and Yamaguchi-Shinozaki, 2007), knowledge of the molecularresponse mechanisms in plants under harsh conditions such as drought is still limited. The understanding of how the expression of individual genes contributes to the final response in cellular, physiological and agronomical terms would enable the design of strategies to obtain plants more tolerant to water deficits (Stolf-Moreira et al., 2010). In this sense transcription factors are powerful tools as they are proteins that interact with cis-elements present in the promoter region of various abiotic stress-related genes thus regulating their expression.

Stolf-Moreira et al. (2011a) working with microarray showed the differential expression of 145 genes in soybean roots under drought stress that were categorized according to their biological roles as genes involved in energy production, transcription factors, genes involved in the anabolic and/or catabolic reactions of amino acids, lipids, carbohydrates and photosynthesis, stress responsive genes, genes involved in the synthesis of proteins, paths for cell communication, cell cycle, cell transport and genes with an unknown function. Of these genes, those encoding the transcription factors BZIP50, C2H2, MYBJ7 and NAC2 were chosen for the present study which aimed:

To identify consensus sequence that could categorize the transcription factors BZIP50, C2H2, MYBJ7 and NAC2 as members of the gene families bzip, c2h2, myb and nac respectively.

To investigate the evolutionary relationship of BZIP50, C2H2, MYBJ7 and NAC2 transcription factors involved in the stress responses.

To analyze the levels of expression of these transcription factors in two soybean genotypes, tolerant (MG/BR46 Conquista) and sensitive (BR16) under drought stress. 


\title{
MATERIAL AND METHODS
}

\begin{abstract}
Alignments
NCBI software (http://www.ncbi.nlm.nih.gov.) was used to find out the predicted amino acids sequences encoded by the BZIP50, C2H2, MYBJ7, and NAC2 transcription factors and these sequences were aligned with sequences of soybean and other plant species using the Clustal X software. Phylogenetic trees were built using the Software Mega 4.1 by Neighbor Joining and the confiability test by Bootstrap.
\end{abstract}

\section{Gene expression analysis}

\section{Plant material and experimental conditions}

Seeds of two genotypes, tolerant (MG/BR46 Conquista) and sensitive (BR16) to drought were germinated in a controlled environment chamber and the four-day-old seedlings were transferred to a hydroponic system containing nutrient solution according to Martins et al. (2008). Each treatment consisted of thirty plants, totaling 450 plants per genotype, considering three biological repetitions, in a randomized complete block design experiment. Drought stress was applied at the V3 stage (second trifoliate leaf completely developed). The plants were subjected to drought for $0,25,50,75$, or $100 \mathrm{~min}$ by removing the roots from the nutrient solution and keeping them in the dark. Roots were collected after each period of exposure for molecular analyses. Temperatures in the greenhouse were controlled at $30^{\circ} \pm 2^{\circ} \mathrm{C}$ during the day and $25^{\circ} \pm 2^{\circ} \mathrm{C}$ at night, with relative humidity near $50 \%$.

\section{qRT-PCR analysis}

In order to validate microarray data obtained by Stolf-Moreira et al. (2011a), four transcription factors (BZIP50, C2H2, MYBJ7, and NAC2) were chosen for the expression studies. The genes and their accession numbers were obtained from the NCBI similarity search. Gm $\beta$-actin and $r R N A 18 S$ were used as the reference for normalization (Stolf-Moreira et al., 2011b) (Table 1).

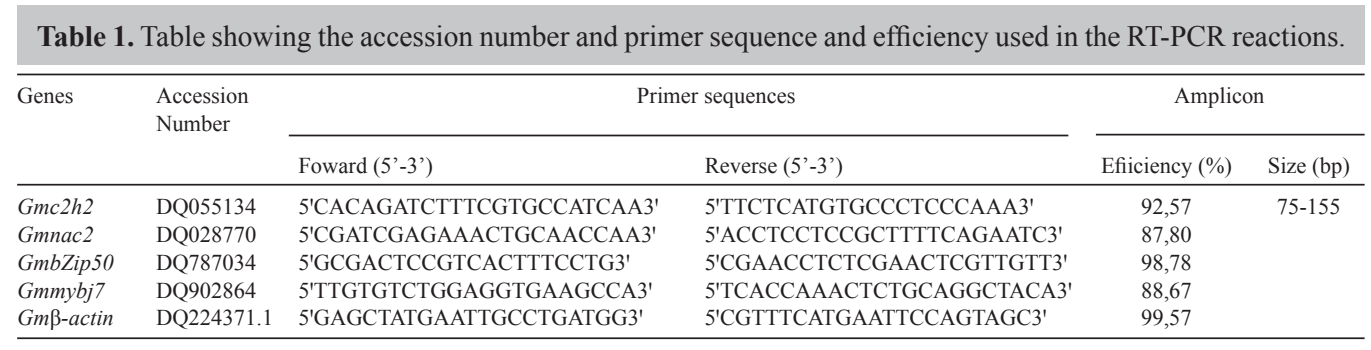

For each period of time, total RNA was extracted using the Trizol reagent (Invitrogen, Carlsbad, USA) according to manufacturer instructions and synthesis of the complementary 
DNA (cDNA) was achieved using the Moloney murine leukemia virus (MMLV) (Invitrogen) reverse transcriptase, according to the method described by Schenck et al. (2003). The Primer Express v.3.0 program (Applied Biosystems) was used to design the primers for qRT-PCR, being determined on the 3 ' regions.

The qRT-PCR analyses were performed using a 7300 Real Time System (Applied Biosystems) thermocycler and the Platinum ${ }^{\circledR} \mathrm{SYBR}^{\circledR}$ Green qPCR SuperMix UDG (Invitrogen). The reaction conditions were $50^{\circ} \mathrm{C}$ for $2 \mathrm{~min}, 95^{\circ} \mathrm{C}$ for $10 \mathrm{~min}$, forty-five cycles at $95^{\circ} \mathrm{C}$ for $2 \mathrm{~min}$, $62^{\circ} \mathrm{C}$ for $30 \mathrm{~s}$ and $72^{\circ} \mathrm{C}$ for $30 \mathrm{~s}$; the data were collected in the last phase (extension phase). The formula $\mathrm{E}=\left[10^{-1 / 1 / \mathrm{lope}}\right]-1$ was used to calculate the reaction efficiency. The results were captured by the Sequence Detection program (Perkin Elmer). Levels of expression of target genes were determined through the using the $2^{-\Delta \Delta C(t)}$ method according to Livac and Schmittgen (2001).

\section{RESULTS}

\section{Alignments}

Sequence alignments of the predicted amino acids encoded by the genes mybj7, nac2, bzip50, and $c 2 h 2$ with other members of the myb, nac, bzip, and $c 2 h 2$ families of soybean and several plant species is shown on Figures 1 to 7 . As consensus sequences (indicated by the asterisk) were identified, these genes were categorized as member of the myb, nac, bzip, and $c 2 h 2$ families respectively. High similarity was found comparing the sequences of the mybj7 gene with other members of the $m y b$ family of soybean. By searching at the NCBI database it was found that the number of members of this family is quite high meaning that they participate in several plant processes. The $m y b$ family is one of the largest families of genes which have the most numbers and functions in plants (Du et al., 2009). The results of phylogenetic analysis revealed that two large groups were formed by the $m y b$ family and that the $m y b j 7$ gene showed similarity with the myb78 of soybean (Figure 8). Other MYB classes were also found in soybean.

Only a few similarities were found comparing the predicted amino acid sequences encoded by the soybean's bzip50 gene with the amino acid sequences encoded by the genes bzip of other plant species. It was confirmed that members of the bzip family are very distinct. The conserved domain characterizing the bzip 50 as belonging to this family was only identified when the sequences were compared to sequences of other members of the soybean's bzip family. The genes used in the alignments were separated in two different groups in the phylogenetic tree (Figure 8). This can be explained by the fact that only the conserved domain was common among these genes. The bzip50 closest related gene was the soybean bzip48 (Figure 8).

A conserved domain QALGGH specific of plants was found by comparing the soybean's $c 2 h 2$ gene with other plant species such as found by Agarwal et al. (2007). By examining the phylogenetic tree built using all the data of the $c 2 h 2$ alignments, it was found that this gene was most closely related to the $c 2 h 2$ of Brassica napus, thus confirming that genes of this family are very diverse among them (Agarwal et al. 2007). Several zinc finger proteins containing a DLN-box/EAR-motif have exhibited transcription repressive activities by transient analysis in plants, such as petunia ZPT2-3 (Sugano et al., 2003) and Arabidopsis STZ/ ZAT10 (Sakamoto et al., 2004). ZFP179 showed transcriptional activation activity in yeast cells although it contains a DLN-box/EAR-motif. Similarly, the zinc finger proteins ThZF1 from salt cress (Xu et al., 2007) and CaZF from chickpea (Jain et al., 2009), both containing 
the DLN-box/EAR-motif, exhibited their transcriptional activation activities. Altogether, it is suggested that some unknown domains may affect the transcriptional activity of $\mathrm{C} 2 \mathrm{H} 2$-type zinc finger proteins (Figure 8).

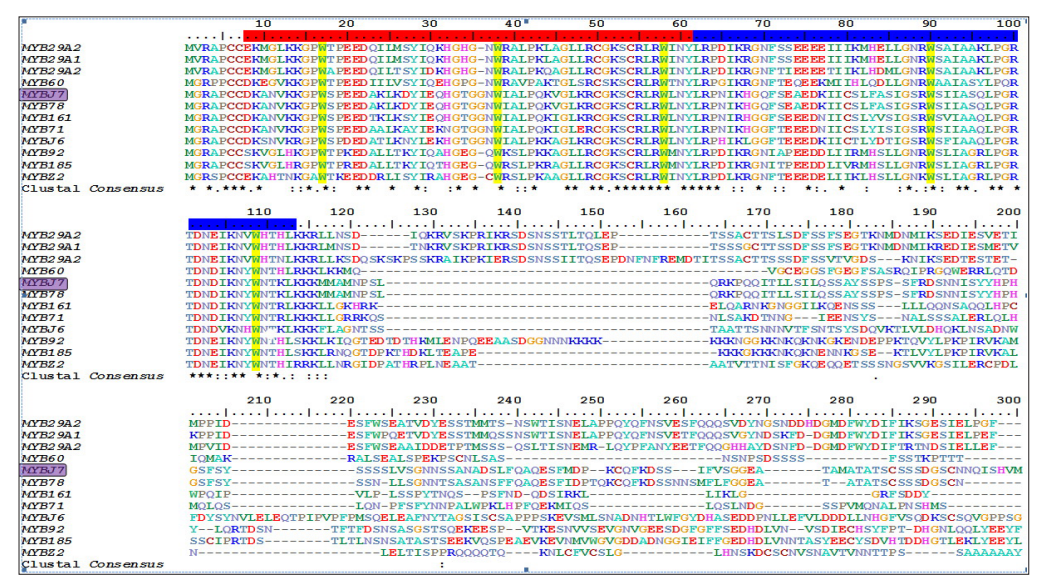

Figure 1. Sequence alignment's of the predicted amino acid sequence encoded by the soybean's mybj7 gene (accession number ABI73973.1) with sequences of other members of the soybean's myb family: myb29a2 (accession number: BAA81732.1); myb29al (accession number: BAA81730.1); myb29a2 (accession number: BAA81733.2); myb60 accession number: ABH02908.1); myb78 (accession number: ABH02914.1); myb161 (accession number: ABH02906.1); myb71 (accession number: ABH02912.1); mybj6 (accession number: ABI73972.1); myb92 (accession number: ABH02844.1); myb185 (accession number: ABH02841.1) e mybz2 (accession number: ABI73970.1). Consensus sequences are identified by an asterisk (*). The red, blue and yellow traces indicate the R2 and R3 domains and the tryptophan regularly spaced respectively.

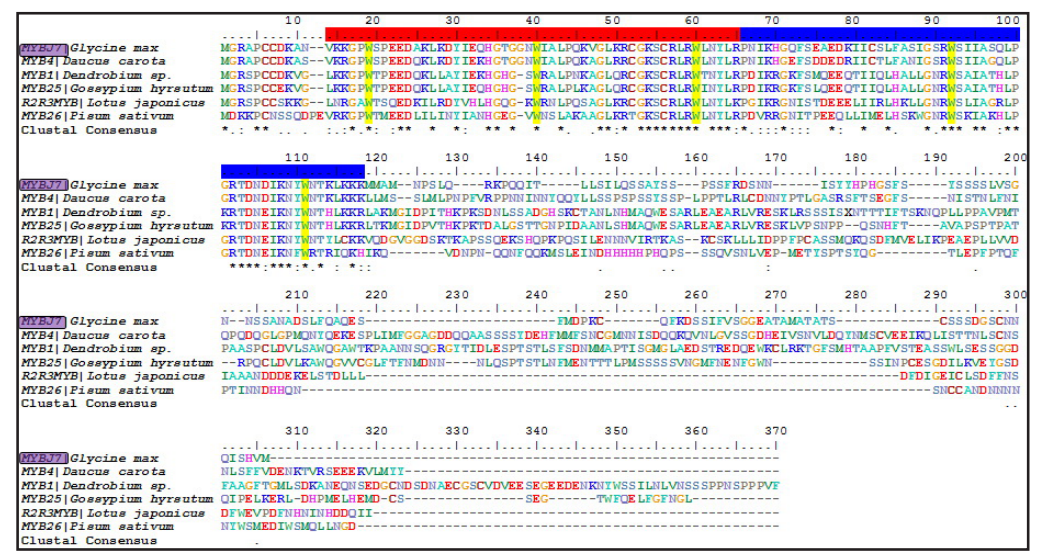

Figure 2. Sequence alignment's of the predicted amino acid sequence encoded by the soybean's mybj7 gene (accession number ABI73973.1) with sequences encoded by the genes myb4 of Daucus carota (accession number: BAF49444.1); myb1 of Dendrobium sp. (accession number: AF485892_1); myb25 of Gossypium hirsutum (accession number: AF336283 1), r2r3myb of Lotus japonicas (accession number: BAG12895.1) and myb26 of Pisum sativum (accession number: CAA71992.1). Consensus sequences are identified by an asterisk (*). The red, blue and yellow traces indicate the R2 and R3 domains and the tryptophan regularly spaced respectively. 

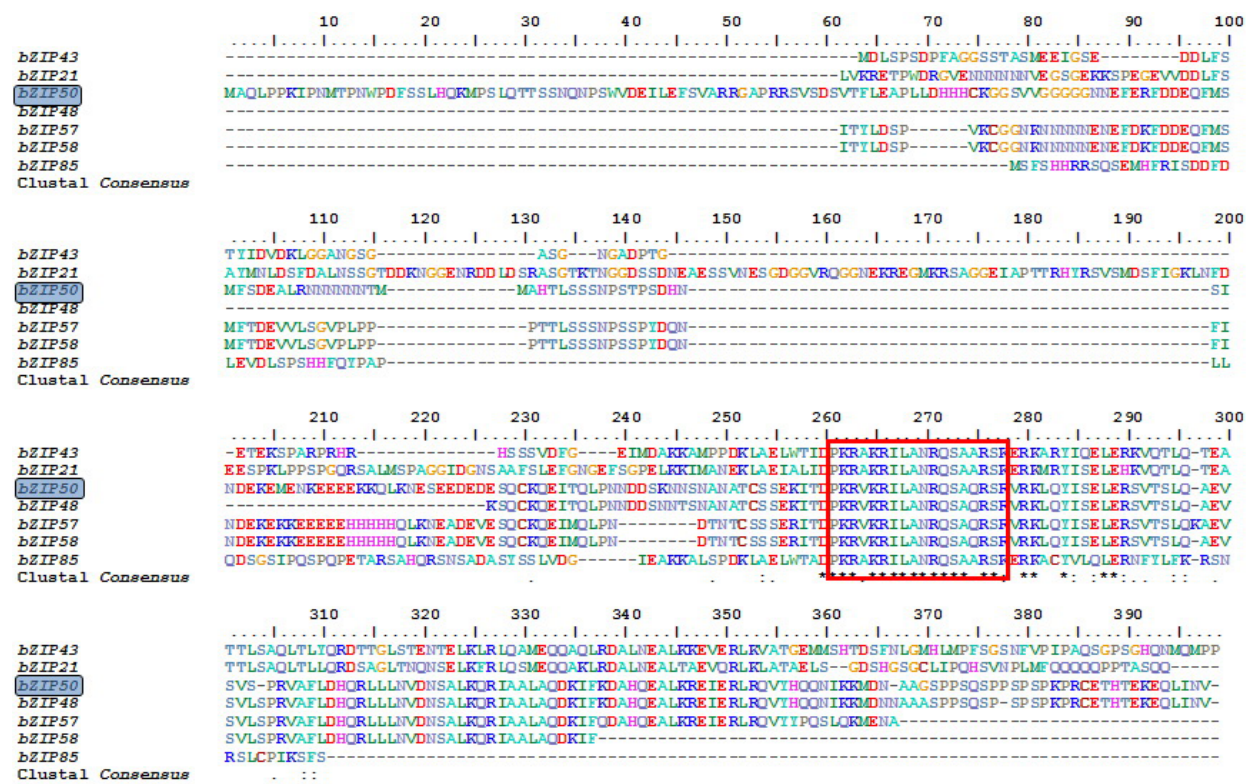

Figure 3. Sequence alignment's of the predicted amino acid sequence encoded by the soybean's bzip50 gene (accession number: ABI34644.1) with sequences of other members of the soybean's bzip Family: bzip43 (accession number: ABI34685.1); bzip 21 (accession number: ABI34695.1); bzip48 (accession number: ABP88222.1); bzip57 (accession number: ABI34683.1); bzip58 (accession number: ABP88224.1) and bzip85 (accession number: ABP88224.1). Consensus sequences are identified by an asterisk (*). The bzip domain is in the red square.

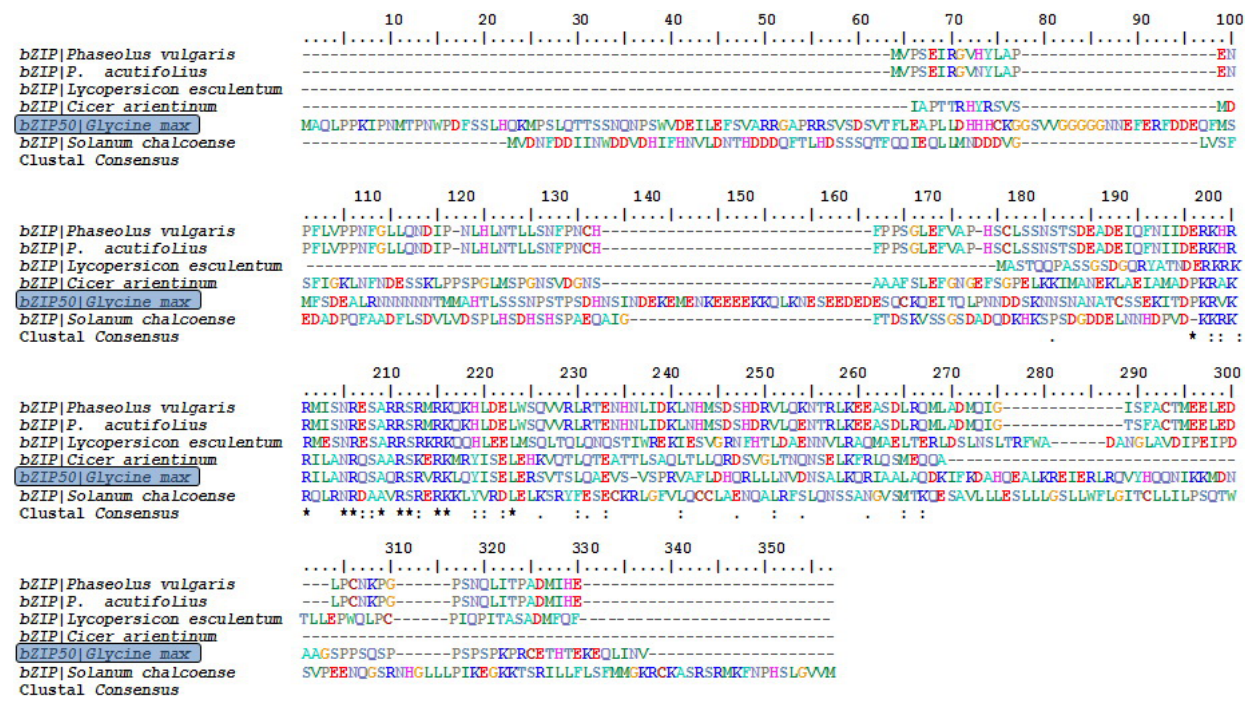

Figure 4. Sequence alignment's of the predicted amino acid sequence encoded by the soybean's bzip50 gene (accession number: ABI34644.1) with sequences encoded by the genes bzip of Phaseolus vulgaris (accession number: AF350505_1); P. acutifolius (accession number: AAK01953.1); Cicer arietinum (accession number: BAA76425.1); Lycopersicon sculentum (accession number: AAD55394.1); and Solanum chacoense (accession number: ACB32232.1). Consensus sequences are identified by an asterisk (*). 


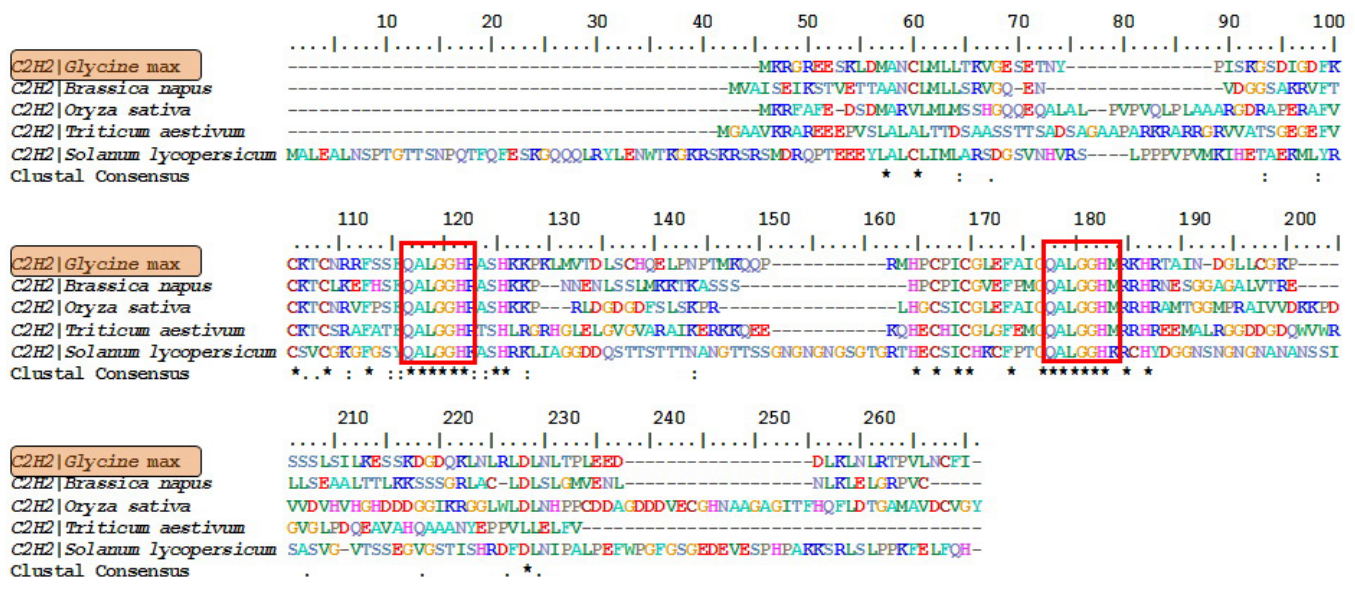

Figure 5. Sequence alignment's of the predicted amino acid sequence encoded by the soybean's $c 2 h 2$ gene (accession number: abq53139.1) with sequences encoded by the genes $c 2 h 2$ of Brassica napus (accession number: abb52061.1), Oryza sativa (accession number: aap74357.1), Triticum aestivum (accession number: abz80834.1) e $S$. lycopersicum (accession number: abr68563.1). Consensus sequences are identified by an asterisk. The $c 2 h 2$ specific domain is in the red square.

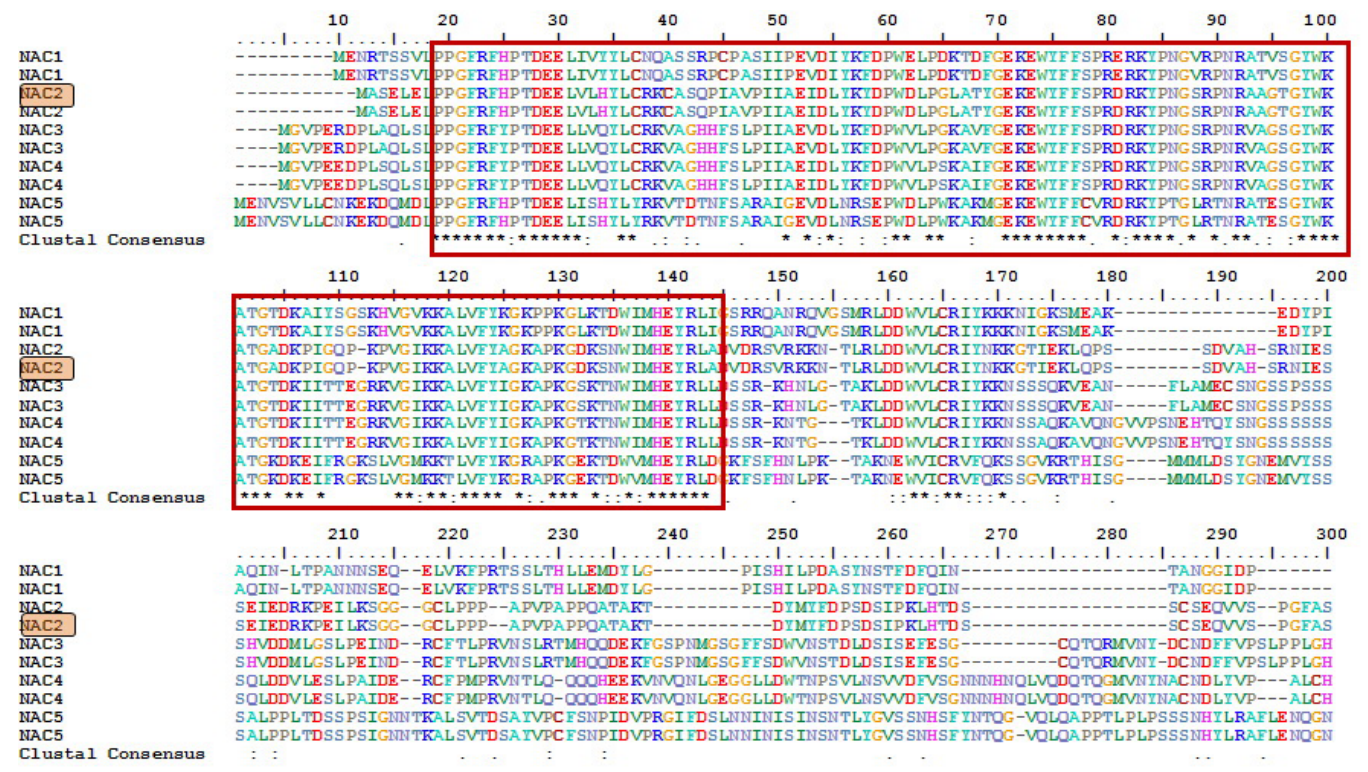

Figure 6. Sequence alignment's of the predicted amino acid sequence encoded by the soybean's nac 2 gene (accession number; AAY46122.1) with sequences of other members of the soybean's nac family: nac2 (accession number; AAX85979.1); nacl (accession number; AAY46121.1); nacl (accession number; AAX85978.1); nac3 (accession number; AAY46123.1); nac3 (accession number; AAX85980.1); nac4 (accession number; AAX85981.1); nac4 (accession number; AAY46124.1); nac5 (accession number; AAY46125.1); nac5 (accession number; AAX85982.1). Consensus sequences are identified by an asterisk (*). 

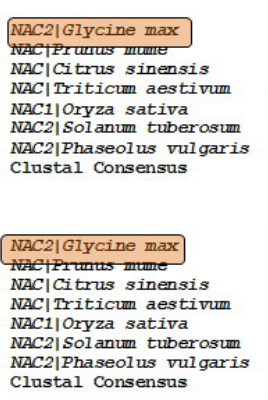
Clustal Consensulgar is

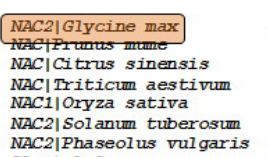
NAC2| Sol anum tuberosum NAC2|Phaseolus vulg
Clustal Consensus

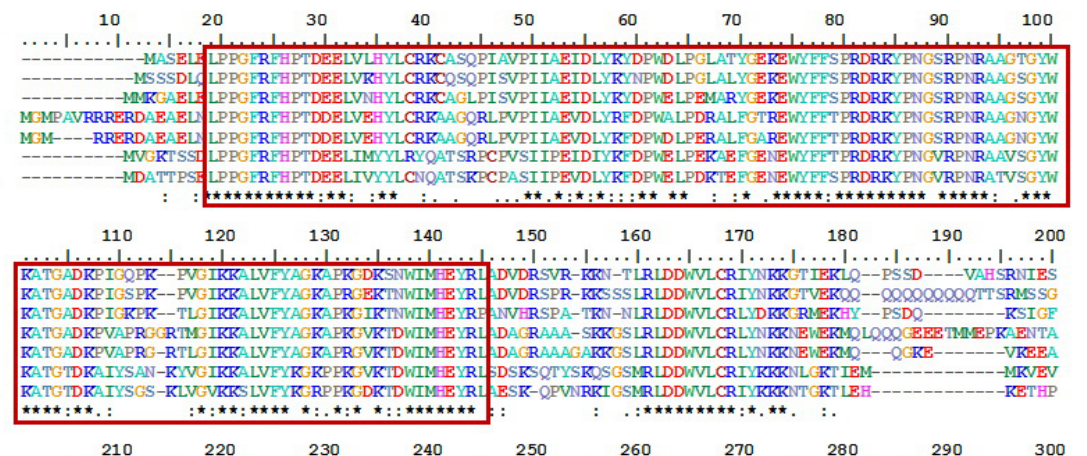

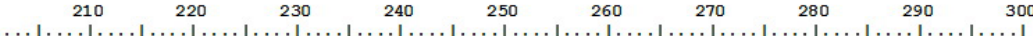

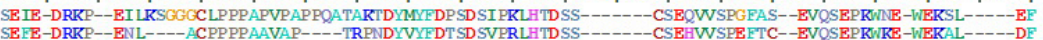

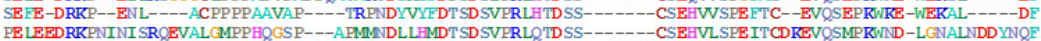
SDMVVT SHSHSQSQSHSHSWGEARTPESE-----TVDNDPSLFQQATAAAEQQAQSP---AAAAAHQEMMATLMNPKKEAADEACRND-LFVDLSYDDIQS

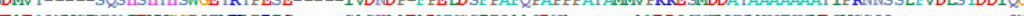

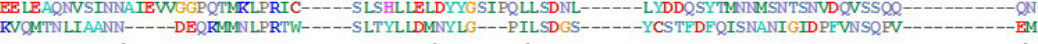

$: \quad:$.

Figure 7. Sequence alignment's of the predicted amino acid sequence encoded by the soybean's nac2 gene (accession number: AAY46122.1) of Prunus mume (accession number: BAE48667.1), Citrus sinesis (accession number: ABQ96643.1), Triticum aestivum (accession number: AAU08786.1), O. sativa (accession number: ABD52007.1), Solanum tuberosum (accession number: ABK96797.1) e P. vulgaris (accession number: AF402603_1). Consensus sequences are identified by an asterisk $(*)$.

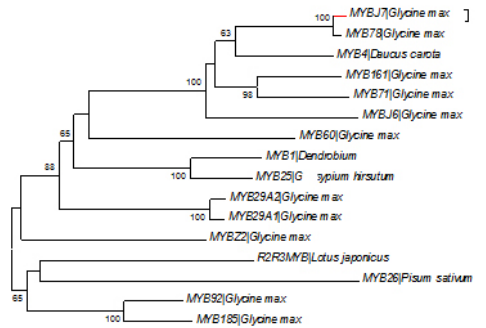

$\stackrel{0.5}{1}$

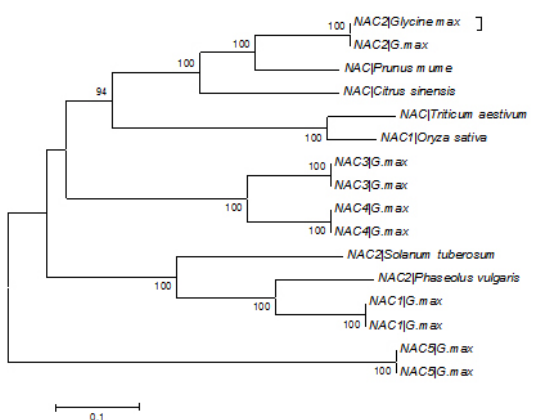

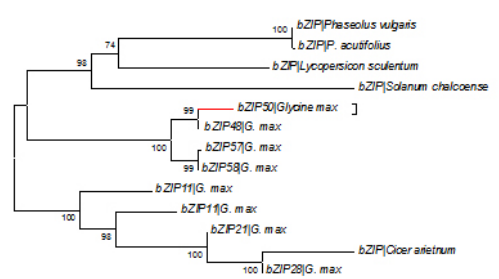

$\longmapsto$

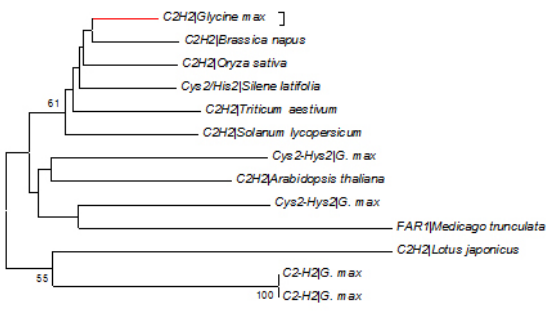

$\longmapsto 0.2$

Figure 8. Phylogenetic tree analysis of predicted amino acid sequences of soybean mybj7, bzip50, c2h2 and nac2 with genes of soybean and other plant species aligned by Clustal X; the tree was generated by Neighbor-Joining method. Branch numbers represent a percentage of the bootstrap values in 1000 sampling replicates and the scale bar indicates branch lengths. 
Members of the $n a c$ family were all grouped except for the nac 5 gene which was isolated. Nac2 of soybean had the nac of Prumus mume as closest gene (Figure 8).

The results of the alignments were used to design the primers for the RT-qPCR. Regions containing less conserved sequences were used in order to prevent amplifications of non target genes as great similarities were found within members of the families studied.

\section{Gene expression analysis}

Expression of the genes was in general increased under drought stress for the two genotypes (Figure 9). Exceptions were found for the mybj7 gene at $75 \mathrm{~min}$ in the MG/BR46 Conquista (tolerant), the bzip50 at $25 \mathrm{~min}$ in the BR16 (sensitive) and for the $c 2 \mathrm{~h} 2$ gene at 100 min in the BR46 Conquista (tolerant).
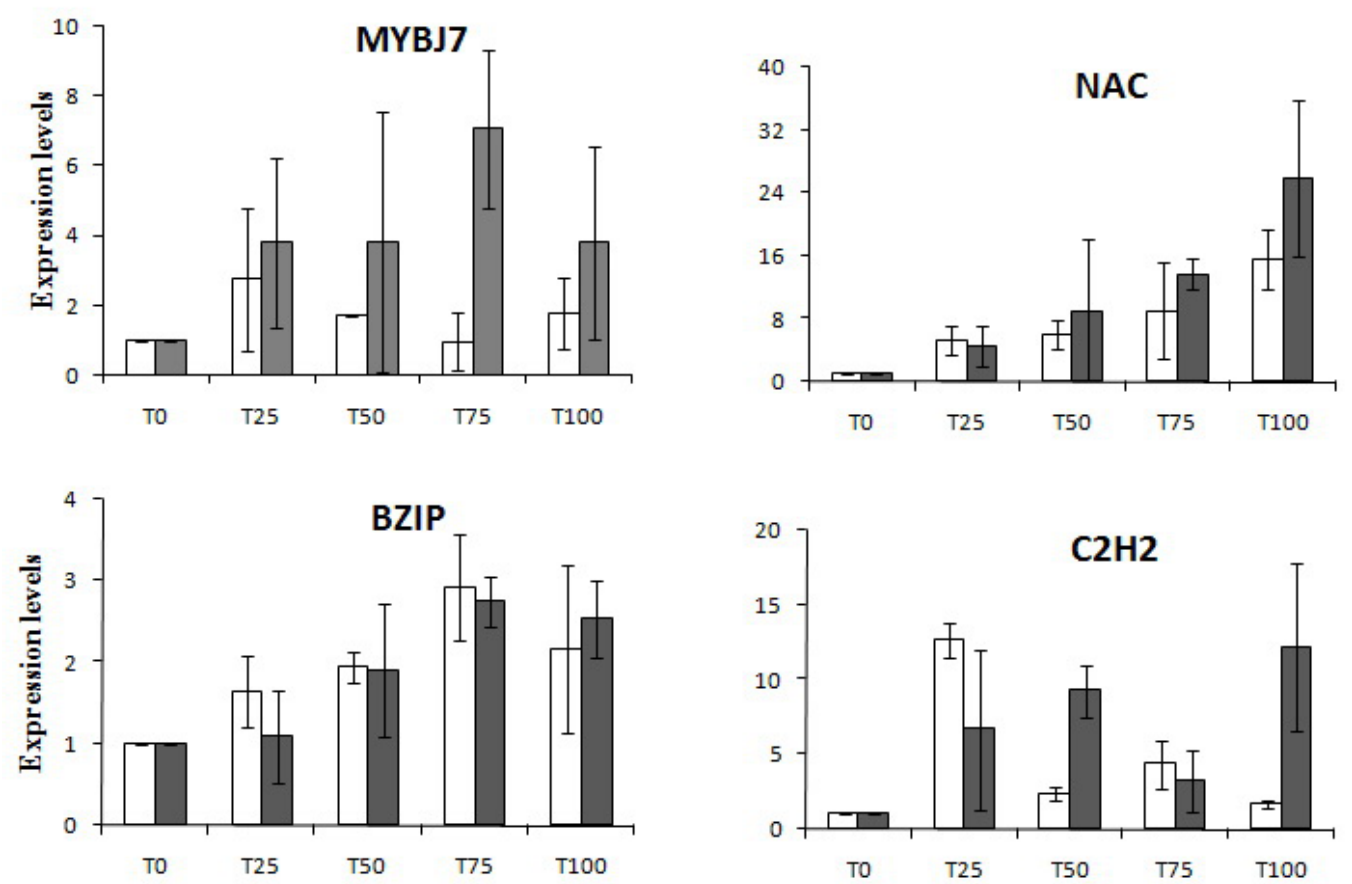

Figure 9. Relative expression of the MYBJ7, bZIP50, NAC2 and $\mathrm{C} 2 \mathrm{H} 2$ transcription factors under drought stress. White and grey lines represent the tolerant (MG/BR46 Conquista) and the sensitive (BR16) genotypes respectively. Vertical bars represent the standard deviation.

Some genes increased their expression over the dehydration period such as the nac2; others increased their expression until $75 \mathrm{~min}$ and then decreased (bzip50). However, others had their expression oscillating between periods of increase and decrease (c2h2). Finally, others such as the mybj7 gene had their expression increased at least until 75 min for one genotype-BR16 (sensitive) and decreased for the other genotype-MG/BR46 Conquista (tolerant).

Major differences between genotypes were due to the lowering of the expression of 
the mybj7 gene (about seven times lower) in the MG/BR46 Conquista (tolerant) at 75 min and the $c 2 h 2$ at 50 and 100 min which was four and seven times lower in the MG/BR46 Conquista (tolerant) respectively.

\section{DISCUSSION}

Plant growth is greatly affected by environmental abiotic stresses such as drought, high salinity and low temperature. Substantial efforts have been devoted to determine the nature of the injury caused by these stresses and the plant-protection mechanisms involved in tolerance responses. In this sense stress-induced transcription factors have been considered a powerful tool as they can regulate expression of several genes. Therefore, it became imperative to study the responses of sensitive and tolerant plants to drought at a molecular genetic level.

In our study we confirmed that the transcription factors assessed (MYB, BZIP, C2H2 and NAC) belongs to the $m y b$, bzip, $c 2 h 2$, and $n a c$ families respectively (Figures 1 to 7 ). Based on the phylogenetic trees, closely related genes were identified and evolutionary relationship among transcription factors of soybean and other plant species were established (Figure 8). It was found that $m y b j 7, n a c 2$, bzip 50 were closely related to $m y b j 78, n a c 2$, bzip 48 of soybean and that the $c 2 h 2$ was more closely related to the $c 2 h 2$ of $B$. napus. The results also revealed that two large groups were formed for the myb family members. Also, bzip genes of soybean separated in two different groups showing that only the conserved domain was common among these genes. The $c 2 h 2$ gene was most closely related to the $c 2 h 2$ of $B$. napus, thus confirming that genes of this family are very diverse (Agarwal et al., 2007). Members of the $n a c$ family were all grouped except for the nac5 gene which was isolated.

Structurally transcription factors are usually classified by their DNA-binding domains. MYB transcription factors contain up to three 52-amino acid residue repeats, the R1, R2 and R3 MYB domains. Plant MYB proteins contain one to three MYB domains, but the majority, like the MYBJ7, are R2R3 type (Chen et al., 2005). BZIP transcription factors have a basic region that binds DNA and a leucine zipper dimerization motif (Nijhawan et al., 2008). The NAC domain was originally defined by the highly conserved N-termini of the petunia nam (no apical meristem), Arabidopsis ataf1 and cuc2 (cup-shaped cotyledon2) genes (Olsen et al., 2005). The classical $\mathrm{C} 2 \mathrm{H} 2$ zinc finger typically contains a repeated 28-30 amino acid sequence, including two conserved cysteines and two conserved histidine residues (Agarwal et al., 2007).

Drought stress affects $m y b j 7$, bzip, $c 2 h 2$, and $n a c 2$ genes and its expression increased for both the tolerant MG/BR46 and sensitive BR16 genotypes (Figure 9). Differences between the two genotypes were mainly due to diminished expression of the mybj7 gene (about seven times lower) in the MG/BR46 Conquista (tolerant) at $75 \mathrm{~min}$ and the $c 2 \mathrm{~h} 2$ at 50 and $100 \mathrm{~min}$ (four and seven times lower respectively).

Most MYB proteins function as transcription factors although many other roles have been attributed to them. According to the literature they have been involved in hormone response, growth, epidermal cell fate and formation of trichomes, stomatal movements and development seed development, response to drought and cold, pathogen-response, light-sensing responses, sugar-related responses, modulation of secondary metabolites such as glucosinolates and synthesis of specific classes of phenylpropanoids such as flavonoids, anthocyanin and lignins (Wilkins et al., 2009; Dubos et al., 2010).

Some of the plant processes that are affected by the expression of MYB tran- 
scription factors may confer drought tolerance. For example, reduction in lignin content which is a phenylpropanoid compound may promote growing of the roots. On the other hand, increases in lignin content may reinforce cell walls for mechanical strength and for water conductance (Andrade, 2006; Fan et al., 2006; dos Santos et al., 2008). These are both desirable characteristics for acquisition of drought stress tolerance depending on when the changes in lignifications occur. Yoshimura et al. (2008) showed that when lignin is synthesized later in the dehydration period it may increase the mechanical strength of the root tissues favoring rooting in to the dry layers of the soil. Fornale et al. (2006) showed that over expression of ZmMYB31 and ZmMYB42 attenuates expression of genes related to lignin synthesis. Aspen (Populus tremuloides Michx.) with reduced expression of the lignin biosynthetic Pt4CL1 gene exhibited up to $45 \%$ reduction in lignin and substantially enhanced cellulose synthesis as well as increased leaf, root, and stem growth (Hu et al. 1999).

Another gene that had its expression decreased in the tolerant MG/BR46 Conquista was the $c 2 h 2$ gene at 50 and 100 min of dehydration. $\mathrm{C} 2 \mathrm{H} 2$ are zinc finger proteins has been involved in a variety of processes such as the regulation of floral organogenesis, leaf initiation, lateral shoot initiation, gametogeneses (Takatsuji, 1999) and stress response. Several studies have reported that overexpression of some $\mathrm{C} 2 \mathrm{H} 2$-type zinc finger protein genes resulted in both the activation of some stress related genes and enhanced tolerance to salt, dehydration, and/or cold stresses (Sakamoto et al., 2000, 2004; Kim et al., 2001; Sugano et al., 2003, Xu et al., 2007).

The other two genes studied bzip and nac2 did not express differentially in the two genotypes but were all increased under drought stress. In plants, basic region leucine zipper motif bzip transcription factors regulate processes including pathogen defense, signaling, seed maturation and flower development (Jakoby et al., 2002). Over expression of ZFP 179 increased proline and soluble sugars contents, enhanced the reactive oxygen species scavenging ability and increased the expression of a number of stressrelated genes, including OsDREB2A, OsP5CS OsProT, and OsLea3 under salt stress (Sun et al., 2010).

Roles of the nac family genes include embryo and shoot meristem development, lateral root formation, auxin signaling, defense and abiotic stress response (Olsen et al., 2005; Kunieda et al., 2008). Guo and Gan (2006) showed that expression of AtNAP, one of the nac family transcription factor genes is associated with leaf senescence.

Under salt stress conditions, the stress inducible expression of the activated Atbzip17 enhanced salt tolerance as demonstrated by chlorophyll bleaching and seedling survival (Liu et al., 2008). Expression of StZFP1 can be induced by salt, dehydration, exogenously applied ABA and to infection by the Phytophthora infestans. These results demonstrate that StZFP1 might be involved in potato responses to salt and dehydration stresses through an ABA-dependent pathway (Tian et al., 2010).

Plant adaptive responses to drought are coordinated by adjusting growth and developmental processes as well as molecular and cellular activities. The root system is the primary site that perceives signals and its development is profoundly affected by the water content (Seo et al., 2009).

Our results indicate that the genes $m y b, b z i p, c 2 h 2$, and $n a c$ are likely to play important roles in roots as they belong to families which are enriched with members that are potentially involved in regulating cellular activities during the changes that occur under drought stress. 


\section{ACKNOWLEDGMENTS}

We thank Embrapa Soybean for the use of the laboratory and greenhouse and CAPES for the financial assistance.

\section{REFERENCES}

Agarwal P, Arora R, Ray S, Singh AK, et al. (2007). Genome-wide identification of $C 2 H 2$ zinc-finger gene family in rice and their phylogeny and expression analysis. Plant Mol. Biol. 65: 467-485.

Andrade AB (2006). Inibição do Crescimento de Raízes de Soja pela Mimosina: Lignificação e Enzimas Relacionadas. Thesis, Universidade Estadual de Maringá, Maringá.

Chen BJ, Wang Y, Hu YL, Wu Q, et al. (2005). Cloning and characterization of a drought-inducible MYB gene from Boea crassifolia. Plant Sci. 168: 493-500.

Conab - Companhia Nacional de Abastecimento (2005). Available at [http://www.conab.gov.br]. Accessed..........

dos Santos WD, Ferrarese ML, Nakamura CV, Mourao KS, et al. (2008). Soybean (Glycine max) root lignification induced by ferulic acid. The possible mode of action. J. Chem. Ecol. 34: 1230-1241.

Du H, Zhang L, Liu L, Tang XF, et al. (2009). Biochemical and molecular characterization of plant MYB transcription factor family. Biochemistry 74: 1-11.

Dubos C, Stracke R, Grotewold E, Weisshaar B, et al. (2010). MYB transcription factors in Arabidopsis. Trends Plant Sci. 15: 573-581.

Embrapa - Empresa Brasileira de Pesquisa Agropecuária (2004). Available at [http://www.cnpso.embrapa.br]. Accessed.........

Fan L, Linker R, Gepstein S, Tanimoto E, et al. (2006). Progressive inhibition by water deficit of cell wall extensibility and growth along the elongation zone of maize roots is related to increased lignin metabolism and progressive stelar accumulation of wall phenolics. Plant Physiol. 140: 603-612.

Fornale S, Sonbol FM, Maes T, Capellades M, et al. (2006). Down-regulation of the maize and Arabidopsis thaliana caffeic acid O-methyl-transferase genes by two new maize R2R3-MYB transcription factors. Plant Mol. Biol. 62: 809-823.

Guo Y and Gan S (2006). AtNAP, a NAC family transcription factor, has an important role in leaf senescence. Plant $J$. 46: 601-612.

Hu Wen-Jing, Harding SA, Lung J, Popko JL, et al. (1999). Repression of lignin biosynthesis promotes cellulose accumulation and growth in transgenic trees. Nat. Biotechnol. 17: 808-812.

Jain D, Roy N and Chattopadhyay D (2009). CaZF, a plant transcription factor functions through and parallel to HOG and calcineurin pathways in Saccharomyces cerevisiae to provide osmotolerance. PLoS One 4: e5154.

Jakoby M, Weisshaar B, Droge-Laser W, Vicente-Carbajosa J, et al. (2002). bZIP transcription factors in Arabidopsis. Trends Plant Sci. 7: 106-111.

Kizis D, Lumbreras V and Pagès M (2001). Role of AP2/EREBP transcription factors in gene regulation during abiotic stress. FEBS Lett. 498: 187-189.

Kunieda T, Mitsuda N, Ohme-Takagi M, Takeda S, et al. (2008). NAC family proteins NARS1/NAC2 and NARS2/NAM in the outer integument regulate embryogenesis in Arabidopsis. Plant Cell 20: 2631-2642.

Liu JX, Srivastava R and Howell SH (2008). Stress-induced expression of an activated form of AtbZIP17 provides protection from salt stress in Arabidopsis. Plant Cell Environ. 31: 1735-1743.

Livak KJ and Schmittgen TD (2001). Analysis of relative gene expression data using real-time quantitative PCR and the 2(-Delta Delta C(T)) Method. Methods 25: 402-408.

Nijhawan A, Jain M, Tyagi AK and Khurana JP (2008). Genomic survey and gene expression analysis of the basic leucine zipper transcription factor family in rice. Plant Physiol. 146: 333-350.

Olsen AN, Ernst HA, Leggio LL and Skriver K (2005). NAC transcription factors: structurally distinct, functionally diverse. Trends Plant Sci. 10: 79-87.

Sakamoto H, Maruyama K, Sakuma Y, Meshi T, et al. (2004). Arabidopsis Cys2/His2-type zinc-finger proteins function as transcription repressors under drought, cold, and high-salinity stress conditions. Plant Physiol. 136: 2734-2746.

Schenk PM, Kazan K, Manners JM, Anderson JP, et al. (2003). Systemic gene expression in Arabidopsis during an incompatible interaction with Alternaria brassicicola. Plant Physiol. 132: 999-1010.

Seo PJ, Xiang F, Qiao M, Park JY, et al. (2009). The MYB96 transcription factor mediates abscisic acid signaling during drought stress response in Arabidopsis. Plant Physiol. 151: 275-289. 
Shinozaki K (2004) Arabidopsis Cys2/His2-Type zinc-finger proteins function as transcription repressors under drought, cold, and high-salinity stress conditions. Plant Physiol. 136: 2734-2746.

Shinozaki K and Yamaguchi-Shinozaki K (2007). Gene networks involved in drought stress response and tolerance. $J$. Exp. Bot. 58: 221-227.

Stolf-Moreira R, Lemos EGM, Carareto AL, Marcondes J, et al. (2011a). Transcriptional profiles of roots of different soybean genotypes subjected to drought stress. Plant Mol. Biol. Rep. 29: 19-34.

Stolf-Moreira R, Lemos EGM, Abdelnoor RV, Beneventi MA, et al. (2011b). Identification of reference genes for expression analysis by real-time quantitative PCR in drought-stressed soybean. Pesq. Agropec. Bras. 46: 58-65.

Sugano S, Kaminaka H, Rybka Z, Catala R, et al. (2003). Stress-responsive zinc finger gene ZPT2-3 plays a role in drought tolerance in petunia. Plant J. 36: 830-841.

Sun SJ, Guo SQ, Yang X, Bao YM, et al. (2010). Functional analysis of a novel Cys2/His2-type zinc finger protein involved in salt tolerance in rice. J. Exp. Bot. 61: 2807-2818.

Takatsuji H (1999). Zinc-finger proteins: the classical zinc finger emerges in contemporary plant science. Plant Mol. Biol. 39: 1073-1078.

Tian ZD, Zhang Y, Liu J and Xie CH (2010). Novel potato C2H2-type zinc finger protein gene, StZFP1, which responds to biotic and abiotic stress, plays a role in salt tolerance. Plant Biol. 12: 689-697.

Wilkins O, Nahal H, Foong J, Provart NJ, et al. (2009). Expansion and diversification of the Populus R2R3-MYB family of transcription factors. Plant Physiol. 149: 981-993.

Xu S, Wang X and Chen J (2007). Zinc finger protein 1 (ThZF1) from salt cress (Thellungiella halophila) is a Cys-2/His2-type transcription factor involved in drought and salt stress. Plant Cell Rep. 26: 497-506.

Yoshimura K, Masuda A, Kuwano M, Yokota A, et al. (2008). Programmed proteome response for drought avoidance/ tolerance in the root of a C3 xerophyte (wild watermelon) under water deficits. Plant Cell Physiol. 49: 226-241. 